\title{
BMJ Open Systematic review of the methodological quality of controlled trials evaluating Chinese herbal medicine in patients with rheumatoid arthritis
}

\author{
Xin Pan, ${ }^{1}$ Maria A Lopez-Olivo, ${ }^{2}$ Juhee Song, ${ }^{3}$ Gregory Pratt, ${ }^{4}$ \\ Maria E Suarez-Almazor ${ }^{2}$
}

To cite: Pan X, LopezOlivo MA, Song J, et al. Systematic review of the methodological quality of controlled trials evaluating Chinese herbal medicine in patients with rheumatoid arthritis. BMJ Open 2017;7: e013242. doi:10.1136/ bmjopen-2016-013242

- Prepublication history and additional material is available. To view please visit the journal (http://dx.doi.org/ 10.1136/bmjopen-2016013242).

Received 29 June 2016 Revised 17 January 2017 Accepted 18 January 2017

\section{CrossMark}

For numbered affiliations see end of article.

Correspondence to Professor Maria E SuarezAlmazor;

msalmazor@mdanderson.org

\section{ABSTRACT}

Objectives: We appraised the methodological and reporting quality of randomised controlled clinical trials (RCTs) evaluating the efficacy and safety of Chinese herbal medicine $(\mathrm{CHM})$ in patients with rheumatoid arthritis (RA).

Design: For this systematic review, electronic databases were searched from inception until June 2015. The search was limited to humans and non-case report studies, but was not limited by language, year of publication or type of publication. Two independent reviewers selected RCTs, evaluating CHM in RA (herbals and decoctions). Descriptive statistics were used to report on risk of bias and their adherence to reporting standards. Multivariable logistic regression analysis was performed to determine study characteristics associated with high or unclear risk of bias.

Results: Out of 2342 unique citations, we selected 119 RCTs including 18919 patients: 10108 patients received CHM alone and 6550 received one of 11 treatment combinations. A high risk of bias was observed across all domains: $21 \%$ had a high risk for selection bias (11\% from sequence generation and $30 \%$ from allocation concealment), $85 \%$ for performance bias, $89 \%$ for detection bias, $4 \%$ for attrition bias and $40 \%$ for reporting bias. In multivariable analysis, fewer authors were associated with selection bias (allocation concealment), performance bias and attrition bias, and earlier year of publication and funding source not reported or disclosed were associated with selection bias (sequence generation). Studies published in nonEnglish language were associated with reporting bias. Poor adherence to recommended reporting standards $(<60 \%$ of the studies not providing sufficient information) was observed in 11 of the 23 sections evaluated.

Limitations: Study quality and data extraction were performed by one reviewer and cross-checked by a second reviewer. Translation to English was performed by one reviewer in $85 \%$ of the included studies.

Conclusions: Studies evaluating CHM often fail to meet expected methodological criteria, and high-quality evidence is lacking.

\section{Strengths and limitations of this study}

- It is the first study appraising methodological quality and adherence to reporting standards specifically in randomised controlled trials (RCTs) evaluating Chinese herbal medicines in patients with rheumatoid arthritis. No other studies have analysed the characteristics associated with a high risk of bias or poor adherence to reporting standards.

- For methodological quality, we used the risk of bias tool, an instrument endorsed by the Cochrane Collaboration to facilitate improved appraisal of evidence. The tool has shown significant correlations with other appraisal tools.

- For reporting adequacy, we used the Consolidated Standards of Reporting Trials (CONSORT) statement which is a minimum set of recommendations for reporting RCTs. It was developed by a team of international experts and is widely used worldwide and encouraged by the leading journals for the reporting of trials (over $50 \%$ of the core medical journals listed in the Abridged Index Medicus on PubMed-eg, BMJ, BMJ Open, New England Journal of Medicine and Journal of the American Medical Association).

- We did not search the Chinese medical databases.

- More than $85 \%$ of the studies were evaluated by the translator and cross-checked by another reviewer using the translation from the first reviewer.

\section{INTRODUCTION}

Rheumatoid arthritis (RA) is one of the leading causes of disability worldwide, affecting $1 \%$ of the world population. According to the Global Burden of Disease 2010 study, years lived with disability from RA increased from 2566000 in 1990 to 3776000 in $2010 .^{1}$ With ageing populations throughout the world, and declines in mortality, the number of people living with RA will increase substantially over coming decades. 
Chinese herbal medicines (CHMs) have been used to treat RA for many years, mostly in Asia. The use of CHMs is generally based on experience and is influenced by a holistic concept of health. ${ }^{2}$ In traditional Chinese medicine (TCM), RA belongs to the category of 'bi syndrome'; that is, it is believed to be caused by attacks of wind, cold, dampness or heat, which cause disharmony between bodily systems. ${ }^{3}{ }^{4}$ Patients are classified as having a particular TCM syndrome according to their symptoms and then treated with CHMs to decrease inflammation by restoring the affected system or to ameliorate the side effects of disease-modifying antirheumatic drugs. ${ }^{5}$ TCM guidelines have been developed by the Chinese government for the diagnosis and treatment of these syndromes. ${ }^{6-8}$

There are numerous CHM preparations for the treatment of RA, including decoctions, whole plants, plant extracts and patented formulas, ${ }^{9-11}$ all of which can be given as a single herb or a mixture of herbs. ${ }^{12}$ Patented CHMs are often offered as chemical preparations, tablets and capsules with simple and convenient dosing schedules and reduced aftertaste. ${ }^{2} 12$ This has increased the acceptance of CHM in western countries. In 2002, a national survey conducted in the USA reported that almost $20 \%$ of adults had used herbal therapies in the past year. ${ }^{13}$ In the UK, a systematic review of 89 surveys on the use of complementary medicine showed that $>50 \%$ of respondents with a chronic condition reported using this type of medicine during their lifetime. ${ }^{14}$ Furthermore, the use rate of herbal therapies in patients with arthritis in US primary care settings has been reported to be as high as $90 \% .^{15}$

After the introduction of evidence-based medicine in China, several randomised controlled trials (RCT) were conducted to evaluate the efficacy of CHMs for RA. ${ }^{10} 12 \quad 16 \quad 17$ However, the methodology used to conduct these trials was inconsistent, and the results were conflicting. Quality of reporting is intrinsically linked to the methodological quality of RCTs. Criteria for standardisation facilitate complete and transparent reporting and help to improve critical appraisal and interpretation of an RCT. Since the development of the Consolidated Standards of Reporting Trials (CONSORT) statement in 1996, most high-impact journals have endorsed its use to improve reporting of RCTs. ${ }^{18}$ In 2006, the CONSORT extension for reporting herbal medicines was developed, and in 2007, a draft of the extension for reporting TCM was released. ${ }^{19}$

To date, no systematic review has explored the study characteristics associated with methodological quality in controlled trials (randomised or not) evaluating the efficacy, effectiveness or safety of CHMs in the treatment of RA. The objectives of our study were to appraise the methodological quality of these studies by ascertaining potential risk of bias, to identify publication factors associated with methodological flaws and to determine the quality of reporting according to CONSORT recommendations.

\section{METHODS}

We report our methods and results according to the Preferred Reporting Items for Systematic Review and Meta-Analysis statement.

\section{Information sources}

We searched electronic databases (Medline, EMBASE, Cochrane Library and Web of Science) from inception through June 2015 for studies evaluating the use of CHM, including herbals or decoctions (eg, 'tang'), in patients with RA (search terms are listed in online supplementary appendix 1). Our search was restricted to human studies and excluded case reports but was not limited by language, year of publication or type of publication. We also searched the reference lists of potentially relevant citations (controlled trials and reviews, although reviews themselves were later excluded from the analysis) to identify additional studies that were not published or otherwise found. EndNote X6 and DistillerSR were used to manage the records retrieved.

\section{Study selection and eligibility criteria}

Two reviewers (XP and PN) independently screened the titles and abstracts of all citations obtained by our searches. They resolved any disagreements through discussion and consensus. When no consensus was reached, a third party acted as an adjudicator (MAL-O). We included any RCT evaluating the efficacy, effectiveness or safety of CHMs in adult patients (age $\geq 18$ years) with RA. All types of CHMs were considered: (1) patented medicines (pharmaceutical preparation or formulations) made from herbs (eg, tablets, liquids, granules, plasters, injections and capsules), (2) herbal decoctions (eg, 'tang') and (3) plants (whole or extracts). Any type of drug and placebo comparison and any follow-up duration were considered for inclusion. We excluded retrieved studies that were published before the year 2000, because the most currently used guidelines for reporting clinical trials (ie, CONSORT statement) were published in 1996, and we conservatively considered 4 years to be enough time for these guidelines to be disseminated and implemented. ${ }^{18}$ We also excluded studies published only as abstracts, studies with a non-RA control group and subanalyses of parent studies.

\section{Data collection and outcome measures}

One author (XP) extracted the data, which were then cross-checked by another author (MAL-O). A standardised extraction form was used to collect information about the characteristics of the RCTs and their participants, types of interventions, reported outcomes and sources of funding. Our primary outcome measures were the methodological and reporting quality of the RCTs.

\section{Methodological quality in individual studies}

The quality of each selected RCT was evaluated independently by two reviewers using the Cochrane risk of bias tool ${ }^{20}$ for RCTs published in English. RCTs published 
in non-English languages were translated and evaluated by one reviewer (XP) and cross-checked by another (MAL-O). In brief, each RCT was evaluated for its potential bias in five domains: selection, performance, detection, attrition and reporting. These domains specifically evaluate how the random sequence was generated, methods of allocation concealment, blinding of participants and personnel, blinding of the outcome assessment, how incomplete outcome data was handled and if there was evidence of selective outcome reporting. Each potential source of bias was graded as low, unclear or high and a justification for each judgement was provided.

\section{Quality of reporting}

We examined how closely the RCTs adhered to reporting standards using the CONSORT statement for $\mathrm{TCM},{ }^{21}$ which focuses only on CHM (ie, acupuncture, moxibustion, cupping and massage are not considered). It is a 23-item checklist, and the major recommendations for transparent reporting are (1) title and abstract should reflect the unique aspects of TCM, (2) rationale of formulation selection should be described, (3) diagnostic criteria should be specified for TCM and conventional medicine, (4) detailed information on the treatment and control interventions should be included, (5) the outcome in TCM terms should be included and (6) the ethics approval number and trial registration number should be included.

\section{Summary measures and synthesis of results}

Descriptive statistics were used to report RCT and participant characteristics, as well as the methodological quality of the RCTs. Risk of bias assessment was summarised per domain. Bivariate analysis was used to compare RCT characteristics according to the risk of bias judgement. Univariate and multivariable logistic regressions were performed to determine the factors associated with high or unclear risk of bias in the five domains in RCTs. We combined the unclear and high risk of bias categories for the analyses. Evidence suggests that the magnitude of treatment effects may be similar in studies appraised as having high or unclear risk of bias, but not for studies assessed as low risk of bias. ${ }^{22}$ The variables tested as predictors were year of publication, sample size, number of authors, publication language (English or non-English), reporting or disclosing of funding (yes or no) and setting (academic or nonacademic). Variables with a univariate $\mathrm{p}<0.15$ were initially included in a multivariable logistic regression model and reduced using the stepwise selection method. Associations were described using ORs and their associated 95\% CIs. We categorised each CONSORT TCM checklist item as reported or not reported. ${ }^{21}{ }^{23}$ We then summarised our findings in five sections: title/abstract, introduction, methods, results and discussion. Subgroup analyses were performed to compare rates of potential bias (low risk or unclear/ high risk, unclear vs high risk) and adherence to reporting guidelines according to (1) publication before or after the Cochrane risk of bias tool was released and (2) publication before or after the CONSORT statement for TCM was released. SAS V.9.3 (SAS Institute, Cary, North Carolina, USA) was used to carry out the computations for all analyses. Apart from the univariate analysis, $\mathrm{p}<0.05$ was considered statistically significant.

\section{RESULTS}

\section{RCT selection}

Out of 2342 unique citations, 232 full-text articles were retrieved to assess for eligibility. Of those, 119 RCTs were included $^{5}{ }^{24-141}$ evaluating 18919 patients. Figure 1 shows the number of studies screened, assessed for eligibility and included in the review, with reasons for exclusion at each stage.

\section{Characteristics of the included studies}

Table 1 shows the aggregated characteristics of the included RCTs, and online supplementary table S1 shows the characteristics of the individual studies. Two-thirds of the RCTs were published before the CONSORT statement for TCM and herbal interventions were published (2000-2007). One RCT was conducted in the USA, one in Korea and the rest in China. Most were single-centre studies and were not indexed in the Web of Knowledge database but were indexed in the China National Knowledge Infrastructure database (CNKI).

Outcomes reported varied across RCTs. More than half of the RCTs assessed efficacy (eg, disease activity score

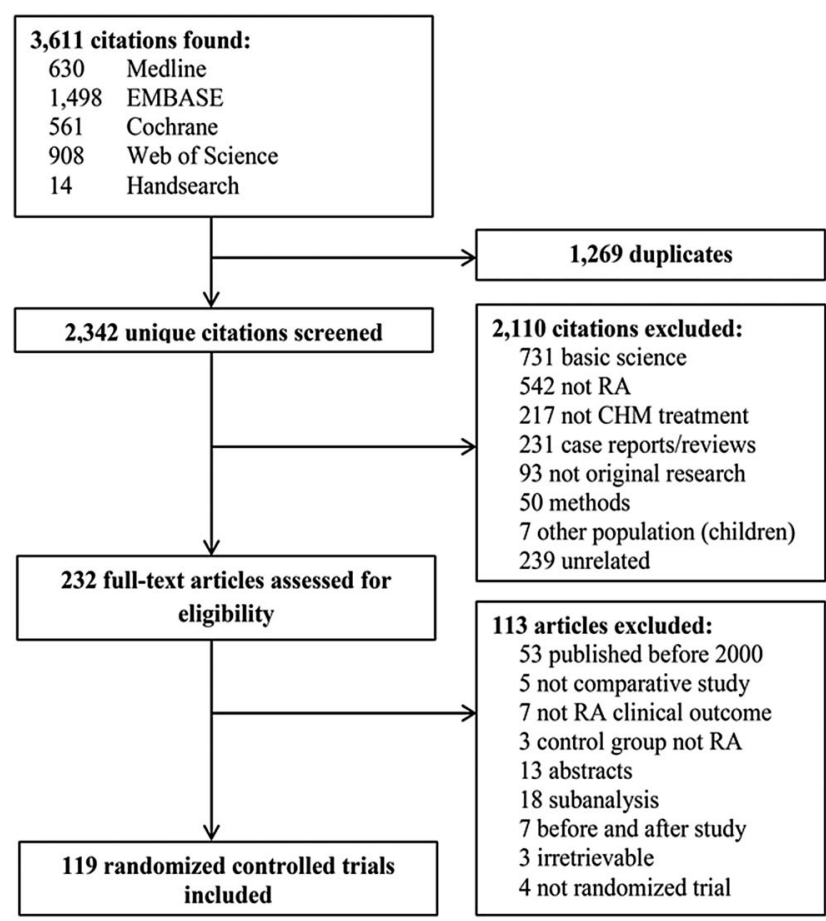

Figure 1 Flow chart of included studies. Abbreviations: RA, rheumatoid arthritis; CHM, Chinese herbal medicine. 
Table 1 Summary of characteristics of the randomised controlled trials (RCTs) included in our analysis $(n=119)$

\begin{tabular}{ll}
\hline RCT characteristic & No. of \\
RCTs (\%)*
\end{tabular}

\section{Duration}

$<6$ months

$93(78)$

$>6$ months

Not reported

$23(19)$

$3(3)$

Year

2000-2007 (before CONSORT

TCM $)^{19} 23142$

2008-2014

Language

English

Chinese

Country

China

$117(98)$

$1(1)$

USA

Scope

Single centre

$108(91)$

Multicentre

Impact factor 2013

Web of Science (score range from

0.59 to 2.711 )

Web of Science (score range from

7.871 to 16.104 )

Indexed in CNKI (score range from

0.031 to 1.068 )

Not indexed $†$

Outcomesł

Disease activity score

ACR 20/30

$15(13)$

$28(24)$

$72(61)$

$78(66)$

$19(16)$

$53(45)$

$66(55)$

$91(76)$

Laboratory outcomes (ESR, CRP, RF or anti-CCP)

Radiographic outcomes

3 (3)

$51(43)$

$76(64)$

$27(23)$

Funding

$\mathrm{CHM}$ preparation (main intervention)

Plant or extract (one herb)

$15(13)$

2 (2)

$52(44)$

Individualised (not following guidelines or

with modifications)

Patented

Patented+individualised

$43(36)$

$15(13)$

Tripterygium wilfordiil Tripterygium

polyglycosides (alone or combined)

Juanbi decoction/mixture or yishen juanbi

capsule (including those with changes or combined)

Feng shi decoction/granules/wine/injection (including those with changes or combined)
Table 1 Continued

\begin{tabular}{lc}
\hline RCT characteristic & $\begin{array}{c}\text { No. of } \\
\text { RCTs (\%)* }\end{array}$ \\
\hline Total glucosides of peony & $6(5)$ \\
Xinfeng capsule & $4(3)$ \\
Zhengqing fengtongning (alone or & $4(3)$ \\
combined) & \\
Bi Qi capsule & $3(3)$ \\
Lugua polypeptide injection & $3(3)$ \\
Guizhi shaoyao zhimu decoction & $3(3)$ \\
Tongbi (tablets or decoction) & $3(3)$ \\
Xiatianwu & $3(3)$ \\
Bizhongxiao & $2(2)$ \\
Sanbi decoction & $2(2)$ \\
Shi re bi granule/mixture & $2(2)$ \\
Siwu wuteng decoction & $2(2)$ \\
Unspecified (including decoctions without a & $11(9)$ \\
name) & \\
\hline
\end{tabular}

${ }^{*}$ Percentages have been rounded.

†Not indexed in Web of Science or CNKI.

\#RCTs can report on one or more outcomes.

§lncluding either clinical safety or laboratory parameters (alanine

transaminase, aspartate transaminase, creatinine, white cell count, platelet count or haemoglobin).

ACR 20/30, American College of Rheumatology criteria for improvement at $20 \%$ or $30 \%$; anti-CCP, anticyclic citrullinated peptide antibodies; CHM, Chinese herbal medicine; CNKI, China National Knowledge Infrastructure database; CONSORT TCM, Consolidated Standards of Reporting Trials statement for traditional Chinese medicine (including herbal interventions); CRP, $C$ reactive protein; $E S R$, erythrocyte sedimentation rate; $R C T$, randomised controlled trial; RF, rheumatoid factor.

either $28{ }^{143}$ or $44 ; ;^{144} 20 \%, 30 \%, 50 \%$ or $70 \%$ improvement, according to American College of Rheumatology criteria, ${ }^{145}$ in tender or swollen joint counts, physician global assessment or grip strength; or good to moderate improvement according to European League Against Rheumatism criteria), ${ }^{146}$ laboratory outcomes (eg, erythrocyte sedimentation rate, $\mathrm{C}$ reactive protein, rheumatoid factor or anticyclic citrullinated peptides) or adverse events. However, $<56 \%$ of RCTs reported on patient-reported outcomes (eg, pain, patient global assessment, health assessment questionnaire or morning stiffness), and only $3 \%$ reported radiographic outcomes (eg, Sharp score, erosion, joint narrowing, marked radiographic progression or no progression). Thirty-one RCTs (26\%) reported improvement of symptoms but did not provide additional details on the type of symptoms or how these symptoms were assessed.

\section{Characteristics of the participants and interventions}

Most participants included in the RCTs met the 1987 American College of Rheumatology (ACR) diagnostic criteria for RA. ${ }^{147}$ One RCT included only patients meeting the 2010 ACR classification criteria ${ }^{148}$ and in another, patients with RCT included met either the 1987 or 2010 criteria. In addition, $52.5 \%$ of the participants met the criteria for one or more of the traditional TCM 'pathological factors' or syndromes (ie, feng 
(wind), shi (damp), tan (phlegm), re (heat) and qi or yin deficiency). The most common pathological factors reported are listed in table 2. A total of 10108 patients received a single CHM and 6550 received one of 11 treatment combinations. Of those receiving combination treatment, 5061 patients received combinations that included CHMs (with disease-modifying antirheumatic drugs, non-steroidal anti-inflammatory drugs, steroids or antibiotics). More than half of the CHMs were individualised preparations targeting pain relief and improvement in joint function. In the control groups, 1402 patients received disease-modifying antirheumatic drugs alone, (ie, methotrexate, leflunomide, sulfasalazine or etanercept), 644 received non-steroidal anti-inflammatory drugs alone and 165 received an inert placebo. In 35 studies, patients were described as having active disease, and two RCTs included patients with refractory disease, one included patients with early RA, three included patients at intermediate stages of RA and one included patients with RA and anaemia. Discontinuation rates were not reported in $68 \mathrm{RCTs}$, but in those that reported discontinuation rates, they ranged from $0 \%$ to $55 \%$.

\section{Quality assessment}

Online supplementary figure S1 summarises the results across RCTs. A high or unclear risk of bias was observed across all domains. When evaluating selection bias, we found that $29 \%$ of the RCTs did not report sufficient detail to evaluate methods of random sequence generation or in $31 \%$ allocation concealment (judged unclear). In addition, $21 \%$ were judged to have high risk for selection bias (11\% from sequence generation, $30 \%$ from allocation concealment). Risk of performance bias (not blinding participants or personnel) was judged to be high in $85 \%$ of the RCTs, and detection bias (blinding of assessment of primary outcome) was judged to be high in $89 \%$ of the RCTs. More than two-thirds of the RCTs did not provide sufficient detail to judge the risk for attrition bias and lacked data on withdrawal rates, power calculation and how missing data was handled. From the remaining RCTs providing details to evaluate attrition bias, $4 \%$ were judged to be of high risk. Risk of reporting bias was high in $40 \%$ of the RCTs, and $86 \%$ of the RCTs did not report the source of funding or included a disclosure statement.

\section{RCT determinants associated with high risk of bias}

Characteristics observed in RCTs according to risk of bias are shown in online supplementary table S2. In the univariate analysis, earlier year of publication, fewer authors, funding source not reported or disclosed, publication in a language other than English, authors from non-academic settings and no power calculation reported were associated with high or unclear risk of bias in various domains (table 3). After adjustment for covariates, the following associations remained in the multivariable analysis: (1) earlier year of publication and funding source not reported or disclosed were
Table 2 Characteristics of the participants in the 119 randomised controlled trials included in our analysis ( $n=18919$, ranging from 30 to 3789 patients per trial)

\begin{tabular}{|c|c|}
\hline Characteristic & No. of patients (\%) \\
\hline Weighted mean age \pm weighted SD & $43.5 \pm 12.0$ years \\
\hline Female & $13697(72.4)$ \\
\hline \multicolumn{2}{|l|}{ Eligibility criteria } \\
\hline ACR RA alone & 8994 (47.5) \\
\hline ACR RA+1 TCM syndrome* & $3040(16.1)$ \\
\hline ACR RA+2 TCM syndromes* & $332(1.8)$ \\
\hline ACR RA+ $\geq 3$ TCM syndromes* & $1072(5.7)$ \\
\hline $\begin{array}{l}\text { ACR RA+unspecified number } \\
\text { TCM syndromes* }\end{array}$ & $300(1.6)$ \\
\hline $\begin{array}{l}\text { ACR RA+TCM syndrome (not } \\
\text { guideline driven) }\end{array}$ & $5181(27.4)$ \\
\hline Median disease duration (range) & $\begin{array}{l}5.4 \text { years } \\
\text { (0.04-28 years) }\end{array}$ \\
\hline \multicolumn{2}{|l|}{ Interventions } \\
\hline CHM alone & $10108(53.4)$ \\
\hline CHM+DMARDs & $1595(8.4)$ \\
\hline CHM+DMARDs+NSAIDs & $511(2.7)$ \\
\hline CHM+DMARDs+steroids+NSAIDs & $79(0.4)$ \\
\hline CHM+steroids & $501(2.6)$ \\
\hline CHM+steroids+NSAIDs & $200(1.1)$ \\
\hline CHM+NSAIDs & $2101(11.1)$ \\
\hline $\mathrm{CHM}+\mathrm{NSAIDs+antibiotic}$ & $74(0.4)$ \\
\hline DMARDs alone & $1402(7.4)$ \\
\hline DMARDs+steroids & $78(0.4)$ \\
\hline DMARDs+steroids+NSAIDs & 363 (1.9) \\
\hline DMARDs+NSAIDs & $992(5.2)$ \\
\hline NSAIDs+antibiotic & $56(0.3)$ \\
\hline NSAIDs alone & $644(3.4)$ \\
\hline Antibiotic $\dagger$ & $50(0.3)$ \\
\hline Placebo alone & $165(0.9)$ \\
\hline Previous WM treatment $\ddagger$ & $8518(45.0)$ \\
\hline \multicolumn{2}{|l|}{ Most common patterns reported§ } \\
\hline Dampness-heat blockage & $1023(10.3)$ \\
\hline $\begin{array}{l}\text { Dampness-cold and qi } \\
\text { deficiency-blood stasis }\end{array}$ & $557(5.6)$ \\
\hline Heat-cold complex & $512(5.2)$ \\
\hline Dampness-cold blockage & $300(3.0)$ \\
\hline $\begin{array}{l}\text { Dampness-heat and qi } \\
\text { deficiency-blood stasis }\end{array}$ & $269(2.7)$ \\
\hline Qi deficiency and blood stasis & $226(2.3)$ \\
\hline Yin deficiency & $178(1.8)$ \\
\hline
\end{tabular}

${ }^{*}$ Chinese medical syndrome following governmental guidelines. †Penicillin.

$\ddagger$ Only 49 studies reported data.

$\S$ Percentages are based on the 46 studies in which eligibility criteria included diagnosis with at least 1 TCM syndrome $(n=9925)$.

ACR RA, American College of Rheumatology classification criteria for rheumatoid arthritis; CHM, Chinese herbal medicine; DMARDs, disease-modifying antirheumatic drugs; NSAIDs, non-steroidal anti-inflammatory drugs; TCM syndrome, traditional Chinese medicine syndrome; WM, western medicine.

associated with high or unclear risk of selection bias (sequence generation); (2) fewer authors were associated with high or unclear risk of selection bias (allocation concealment), performance bias and attrition bias and (3) publication in a language other than English 
Table 3 Characteristics of randomised controlled trials (RCTs) included in our study ( $n=119)$ associated with high or unclear risk of bias

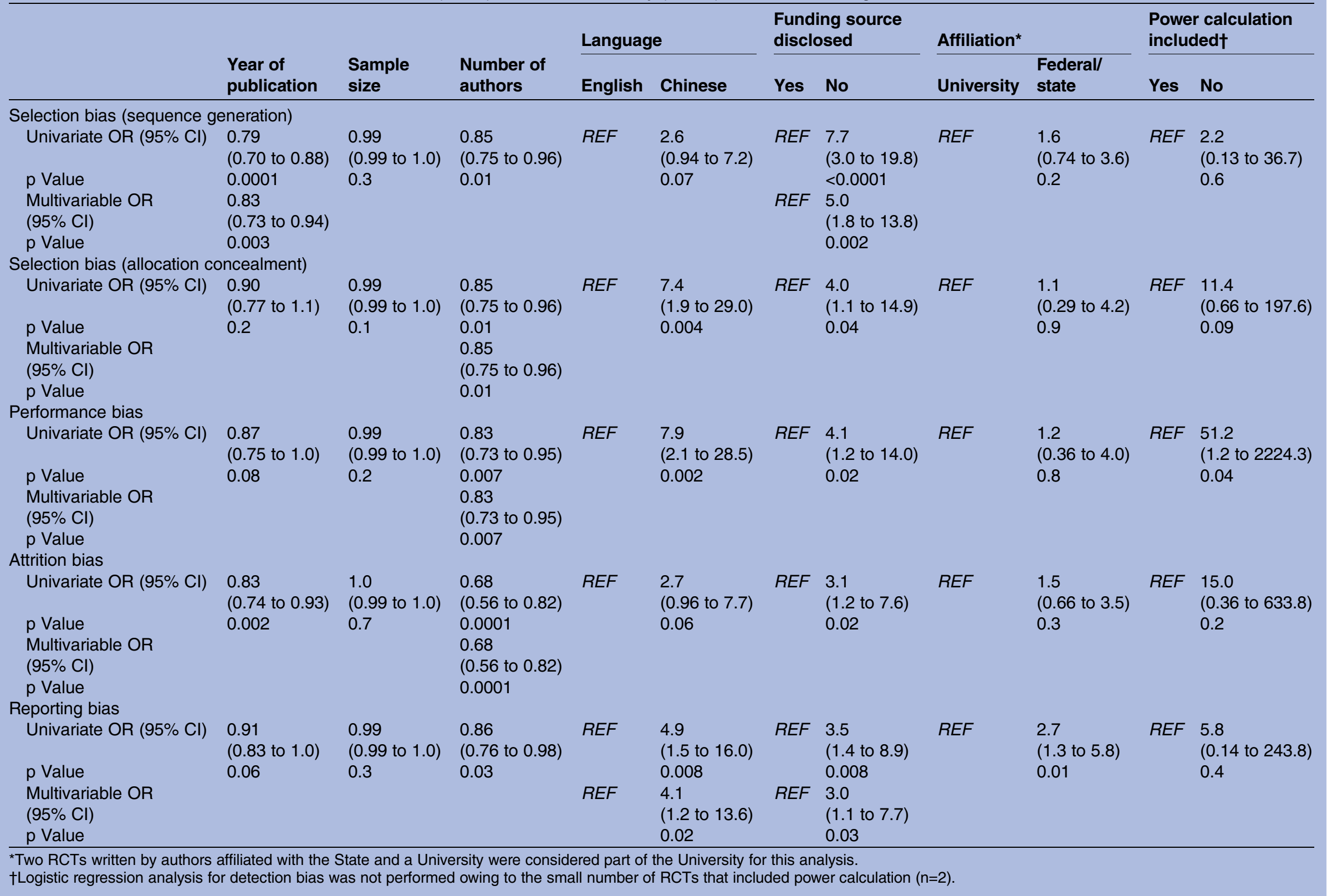


and funding source not reported or disclosed was associated with high or unclear risk of reporting bias. Logistic regression analysis could not be performed for detection bias owing to the small number of RCTs in the low risk-of-bias group $(\mathrm{n}=2)$.

\section{Adherence to CONSORT standards for TCM}

Rates of adherence to CONSORT standards for TCM are shown in table 4 . Most of the RCTs $(98 \%)$ stated the objective adequately. However, most RCTs $(94 \%)$ did not include the recommended information (specification of interventions, name of disease and study design) in the title or abstract. In the introduction sections, more than half $(61 \%)$ did not provide the three names of the compound formulation (Chinese, Latin and English) as recommended by the WHO. The methods sections of most RCTs were poorly reported (table 4). Most RCTs (ranging from 85\% to 98\%) failed to describe in enough detail the interventions, type of study design, calculation of sample size or methods of randomisation and blinding. In the results sections, many RCTs $(78 \%)$ did not indicate how participants moved through the study over time or provide a flow diagram as

Table 4 Percentage of randomised controlled trials in our analysis ( $n=119)$ not adherent to reporting standards provided in the Consolidated Standards of Reporting Trials (CONSORT) statement for traditional Chinese medicine

\begin{tabular}{lcc}
\hline CONSORT item & $\begin{array}{l}\text { CONSORT } \\
\text { item no. }\end{array}$ & No. (\%) \\
\hline $\begin{array}{l}\text { Title and abstract } \\
\text { Introduction }\end{array}$ & 1 & $112(94)$ \\
$\quad$ Background & 2 & $73(61)$ \\
Objectives & 3 & $2(2)$ \\
Methods & & \\
$\quad$ Participants & 4 & $45(38)$ \\
Interventions & 5 & $101(85)$ \\
Outcomes & 6 & $15(13)$ \\
Type of study design & 7 & $117(98)$ \\
Sample size & 8 & $117(98)$ \\
Randomisation & & \\
$\quad$ Sequence generation & 9 & $72(61)$ \\
$\quad$ Allocation concealment & 10 & $109(92)$ \\
$\quad$ Implementation & 11 & $111(93)$ \\
Blinding (blinding) & 12 & $107(90)$ \\
$\quad$ Statistical methods & 13 & $48(40)$ \\
Results & & \\
Participant flow & 14 & $93(78)$ \\
$\quad$ Recruitment & 15 & $33(28)$ \\
$\quad$ Baseline data & 16 & $31(26)$ \\
Numbers analysed & 17 & $111(93)$ \\
Outcomes and estimation & 18 & $11(9)$ \\
Ancillary analyses & 19 & $23(19)$ \\
$\quad$ Adverse events & 20 & $40(34)$ \\
Discussion & & \\
Interpretation & 21 & $9(8)$ \\
Generalisability & 22 & $14(12)$ \\
Overall evidence & 23 & $99(83)$ \\
\hline$\quad$ & &
\end{tabular}

recommended, and 93\% of RCTs did not include intention-to-treat analysis. The discussion sections were compromised in $8-83 \%$ of the RCTs owing to a lack of general interpretation of the results and conflict of interest information.

\section{Additional analyses}

When comparing the risk of bias from the 90 RCTs published before the Cochrane risk of bias tool was released in 2008 (year of publication 2000-2008) with that of the 29 RCTs published later, we observed improvement in RCTs published after 2008 in the following domains: selection bias (22\% compared with $59 \%, \mathrm{p}=0.0004)$, attrition bias ( $18 \%$ compared with $52 \%, \mathrm{p}=0.0005)$ and reporting bias (41\% compared with $66 \%, \mathrm{p}=0.02)$. When comparing adherence to reporting guidelines from the 80 RCTs published before the CONSORT-TCM statement was released in 2007 (publication date 20002007) with that of the 39 RCTs published later, we observed improvement in RCTs published after 2007 in most of the items except in reporting sufficient details about the objectives of the study, design, power calculation and methods to avoid bias (data not shown). We also evaluated determinants associated with high risk of bias, compared with unclear risk of bias. In most domains there were no differences. We observed differences in allocation concealment, but the characteristics associated with high risk of bias were the same as those observed with the main comparison high/unclear versus low risk of bias. There were some characteristics not observed in the main comparison associated with detection bias (see online supplementary table S3).

\section{DISCUSSION}

Our results indicate that serious methodology and reporting flaws still exist in clinical trials evaluating the effect of CHM in RA. We found that the potential for selection bias was high; two-thirds of the RCTs in our analysis lacked sufficient detail on how the random sequence was generated. Similarly, most RCTs were not blinded (eg, for participants, personnel or outcome assessment), thus increasing the potential for performance and detection bias. Risk of attrition bias was high or unclear in more than two-third of the RCTs. Reporting bias was also judged to be high or unclear in half of the included RCTs owing to a lack of study protocol and/or reporting less than the minimum number of outcome measures recommended to be included in RA clinical trials. ${ }^{149}$ Furthermore, we found that RCTs that were older, had fewer authors, did not report or disclose funding, were published in a language other than English or were written by authors from non-academic settings, were likely to have a high or unclear risk of bias methodology in at least one of the evaluated domains.

We also found that adherence to reporting standards remains a concern. Title and abstract, introduction and methods were even more problematic. Owing to these 
weaknesses, readers are not provided with clear and transparent information on the interventions or methods to assess bias in selecting, blinding and evaluating participants. Other areas of concern were lack of appropriate general interpretation of the results in the context of current evidence and incomplete descriptions of conflicts of interest.

It is important to differentiate between the two main concepts reported in this review: methodological quality and quality of reporting. One of the main components of evidence-based medicine is the use of available literature to improve decision-making. Stronger inferences can be drawn from studies in which measures have been taken before, during and after the intervention to prevent random and systematic bias. Although RCTs are ranked high in the hierarchy of evidence, not all RCTs share the same quality, which can lead to biased results. Quality of reporting is a separate concern. A lack of complete and transparent reporting of the processes and findings of an RCT is commonly linked to methodological flaws. Poor reporting leaves out critical information needed to judge study safeguards against bias.

A few systematic reviews evaluating the efficacy of CHM and reporting on the methodological quality of the studies have shown similar results. However, none of these studies evaluated CHMs exclusively (ie, excluding other TCM) in patients with RA. Nonetheless, a review evaluating the characteristics of 89 studies published between 2000 and 2003 and indexed in the CNKI reported a lack of unified diagnostic and evaluating standards. ${ }^{150}$ Another review evaluating 20 RCTs published between 2000 and 2010 showed that methodological quality according to the Jadad scale was generally low, with an average quality score of 1.2 out of $5 .{ }^{151}$ Contrastingly, a Cochrane review evaluating herbal medicines in general (including three CHMs) showed that the quality of the studies has improved since 2000, but the risk of bias across different domains was variable. ${ }^{152}$ Furthermore, an overview of 31 reviews published between 1999 and 2009 evaluating several TCM approaches (including CHMs) for multiple diseases showed that methodological quality improved over the years, although many issues remained, specifically a high risk of selection (inadequate randomisation methods), attrition (small sample sizes and high withdrawal rates) and reporting (selective reporting of outcomes) biases. ${ }^{153}$

To the best of our knowledge, the current study is the first appraising methodological quality and adherence to reporting standards specifically in RCTs evaluating CHM in patients with RA. No other studies have analysed the characteristics associated with a high risk of bias or poor adherence to reporting standards. For methodological quality, we used the risk of bias tool, an instrument endorsed by the Cochrane Collaboration to facilitate improved appraisal of evidence. The tool has shown significant correlations with other appraisal tools. ${ }^{154155}$ For example, the risk of bias tool was shown to accurately identify trials that may have overestimated treatment effects. Studies shown to have a high or unclear risk of bias according to the risk of bias tool have larger effect estimates than studies shown to have a low risk of bias. ${ }^{22}$

Our study has certain limitations. First, as with any systematic review, it was constrained by the available data. For example, a protocol or trial registration was not found for most of the RCTs included in our review. This is concerning because the validity of the conclusion of an RCT is largely based on adherence to prespecified methods (including outcomes of interest). Trial registration could improve transparency and help identify gaps in knowledge, prevent unnecessary duplication in clinical trials and improve adherence to international quality standards. ${ }^{156}$ Second, we included studies with substantial variation in characteristics (eg, RA diagnosis, TCM syndromes and CHM descriptions). However, this allowed us to evaluate a larger number of RCTs. Third, we did not search the CNKI, Chinese Medical Current Contents (CMCC) and Wanfang Data databases, which could have increased the number of publications included in this review, because they are not widely available outside Asia, and we felt that there was no clear reason to consider that the quality of these studies would have been better. ${ }^{157}$ Finally, independent quality assessment for non-English language articles could not be performed. Only one reviewer could translate Chinese articles; therefore, more than $85 \%$ of the studies were evaluated by the translator and cross-checked by another reviewer using the translation from the first reviewer.

In summary, our results indicate that trials of CHM for the treatment of RA often fail to meet expected methodological criteria, and high-quality evidence is lacking. Because clinical trials are just below systematic reviews in the hierarchy of evidence and are used to endorse recommendations by health organisations, more attention is needed to improve the methodological robustness of these studies. Future clinical trials evaluating CHMs in RA should be designed, conducted and reported according to current specifications and principles.

\section{Author affiliations}

${ }^{1}$ Department of Rheumatology, Shuguang Hospital affiliated to Shanghai University of Traditional Chinese Medicine, Shanghai, China

${ }^{2}$ Department of General Internal Medicine, Rheumatology Section, The University of Texas MD Anderson Cancer Center, Houston, Texas, USA ${ }^{3}$ Department of Biostatistics, The University of Texas MD Anderson Cancer Center, Houston, Texas, USA

${ }^{4}$ Research Medical Library, The University of Texas MD Anderson Cancer Center, Houston, Texas, USA

Twitter Follow Maria Suarez-Almazor @msalmazor

Acknowledgements We are grateful to Yimin Geng from the Research Medical Library of The University of Texas MD Anderson Cancer Center for helping with the terms included in the search strategies for the electronic databases and to Ms. Pratibha Nayak for her contributions during the selection of the studies.

Contributors MES-A had full access to all of the data in the study and takes responsibility for the integrity and the accuracy of the data analysis. MES-A and MAL-O conceptualised and designed the study. GP and Geng were responsible for the search strategy. XP, Nayak and MAL-0 were responsible for selection of 
the studies. XP, MAL-0 contributed in quality appraisal and data extraction. JS, XP, MAL- 0 and MES-A analysed and interpreted the data. XP, MAL-0, JS and MES-A drafted the manuscript. XP, MAL-0, JS, GP and MES-A critically revised the manuscript for important intellectual content. MES-A provided administrative, technical or material support and supervised the study.

Funding The statistical analysis in this research (through the Biostatistics Resource Group) was supported in part by a Cancer Center Support Grant from the National Cancer Institute (P30CA016672) to the University of Texas MD Anderson Cancer Center.

Competing interests We have read and understood the BMJ Open policy on declaration of interests and declare the following interests: (1) MES-A was the recipient of a K24 career award from the National Institute for Musculoskeletal and Skin Disorders; (2) XP's work is supported by the Shanghai Municipal Education Commission and the Shanghai Shuguang Hospital, Shanghai University of TCM and (3) MAL- 0 is the recipient of a career award from the Rheumatology Research Foundation and has received a consulting fee from Complete HEOR Solutions outside the scope of the submitted work. All authors have completed the Unified Competing Interest form at http://www. icmje.org/coi_disclosure.pdf (available on request from the corresponding author).

Provenance and peer review Not commissioned; externally peer reviewed.

Data sharing statement No additional data are available.

Open Access This is an Open Access article distributed in accordance with the Creative Commons Attribution Non Commercial (CC BY-NC 4.0) license, which permits others to distribute, remix, adapt, build upon this work noncommercially, and license their derivative works on different terms, provided the original work is properly cited and the use is non-commercial. See: http:// creativecommons.org/licenses/by-nc/4.0/

\section{REFERENCES}

1. Cross $M$, Smith $E$, Hoy D, et al. The global burden of rheumatoid arthritis: estimates from the global burden of disease 2010 study. Ann Rheum Dis 2014;73:1316-22.

2. Zhang C, Jiang M, Lu AP. Evidence-based Chinese medicine for rheumatoid arthritis. $J$ Tradit Chin Med 2011;31:152-7.

3. Li S. Advances in TCM symptomatology of rheumatoid arthritis. $J$ Tradit Chin Med 2002;22:137-42.

4. Tsang IK, Huang SH, Koehler BE. Traditional Chinese medicine: an opportunity for discovery. J Rheumatol 2010;37:1989-90.

5. He YT, Ou AH, Yang XB, et al. Traditional Chinese medicine versus western medicine as used in China in the management of rheumatoid arthritis: a randomized, single-blind, 24-week study. Rheumatol Int 2014;34:1647-55.

6. Wang ZM, Bai RX. Integrated medical diagnosis and treatment criteria for four rheumatoid diseases. Zhong Xi Yi Jie He Za Zhi 1989;9:52-3.

7. Zheng XY. Guideline of clinical research into new Chinese drugs. Vol. 116. Beijing: China Medical Sci-tech Press, 2002.

8. State Administration of Chinese Medicine. Criteria of diagnosis and therapeutic effect of diseases and syndromes in traditional Chinese medicine. Vol. 7. Nanjing University Press, 1994:29-30.

9. Canter PH, Lee HS, Ernst E. A systematic review of randomised clinical trials of Tripterygium wilfordii for rheumatoid arthritis. Phytomedicine 2006;13:371-7.

10. Zhang C, Jiang M, Lu AP. Chinese herbal medicines versus disease modifying antirheumatic drugs for management of rheumatoid arthritis: a systematic review. Eur J Integr Med 2011;3: E219-31.

11. Zhang $P, \mathrm{Li}$ J, Han $Y$, et al. Traditional Chinese medicine in the treatment of rheumatoid arthritis: a general review. Rheumatol Int 2010;30:713-18.

12. Zhang $\mathrm{C}$, Jiang $\mathrm{M}, \mathrm{Guo} \mathrm{H}$, et al. Chapter 7-Chinese herbal medicines for rheumatoid arthritis. In: Lie-Fen S, Allan SYL, eds. Advances in botanical research. Academic Press, 2012:273-313.

13. Manheimer E, Wieland S, Kimbrough E, et al. Evidence from the Cochrane Collaboration for Traditional Chinese Medicine therapies. $J$ Altern Complem Med 2009;15:1001-14.

14. Posadzki P, Watson LK, Alotaibi A, et al. Prevalence of use of complementary and alternative medicine (CAM) by patients/ consumers in the UK: systematic review of surveys. Clin Med (Lond) 2013;13:126-31.

15. Herman CJ, Allen P, Hunt WC, et al. Use of complementary therapies among primary care clinic patients with arthritis. Prev Chronic Dis 2004;1:A12.

16. Chen ZF, Zhang JH. Traditional Chinese herbs for precancerous lesion of esophagus: a meta analysis. Traditi Chin Med Res 1999;12:22-3.

17. Ma B, Guo J, Qi G, et al. Epidemiology, quality and reporting characteristics of systematic reviews of traditional Chinese medicine interventions published in Chinese journals. PLOS ONE 2011;6:e20185

18. Begg C, Cho M, Eastwood S, et al. Improving the quality of reporting of randomized controlled trials. The CONSORT statement. JAMA 1996;276:637-9.

19. Gagnier JJ, Boon $\mathrm{H}$, Rochon $\mathrm{P}$, et al. Reporting randomized, controlled trials of herbal interventions: an elaborated CONSORT statement. Ann Intern Med 2006;144:364-7.

20. Higgins JPT, Green S, eds. Cochrane Handbook for Systematic Reviews of Interventions Version 5.1.0. The Cochrane Collaboration, 2011. www.handbook.cochrane.org

21. Bian Z, Liu B, Moher D, et al. Consolidated standards of reporting trials (CONSORT) for traditional Chinese medicine: current situation and future development. Front Med 2011;5:171-7

22. Hartling $L$, Ospina $M$, Liang $Y$, et al. Risk of bias versus quality assessment of randomised controlled trials: cross sectional study. $B M J$ 2009;339:b4012.

23. Fei YT, Liu JP. [Herbal CONSORT statement and standardization of reporting traditional Chinese drug trials]. Zhongguo Zhong Yao Za Zhi 2008;33:89-94.

24. Chen L, Qi H, Jiang D, et al. The new use of an ancient remedy: a double-blinded randomized study on the treatment of rheumatoid arthritis. Am J Chin Med 2013;41:263-80.

25. Chen Z, Li XP, Li ZJ, et al. Reduced hepatotoxicity by total glucosides of paeony in combination treatment with leflunomide and methotrexate for patients with active rheumatoid arthritis. Int Immunopharmacol 2013;15:474-7.

26. Chen ZW, Sun J, Li YM, et al. [Efficacy of Shenshi Qianghuo Dihuang Decoction in rheumatoid arthritis: a randomized controlled trial]. Zhong Xi Yi Jie He Xue Bao 2010;8:35-9.

27. Du JH, Dong BD. [Comparative study on clinical efficacy of using methotrexate singly or combined with total glucosides of Paeony in treating rheumatoid arthritis]. Zhongguo Zhong Xi Yi Jie He Za Zhi 2005;25:540-2.

28. Fan D-H, Xia B. [Observation on therapeutic effect of heat needle combined with herb iontophoresis and western medicine on rheumatoid arthritis]. Zhongguo zhenjiu 2007;27:731-4.

29. Goldbach-Mansky R, Wilson M, Fleischmann R, et al. Comparison of Tripterygium wilfordii Hook $F$ versus sulfasalazine in the treatment of rheumatoid arthritis: a randomized trial. Ann Intern Med 2009;151:229-40, W49-51.

30. He Y, Lu A, Lu C, et al. Symptom combinations assessed in traditional Chinese medicine and its predictive role in ACR20 efficacy response in rheumatoid arthritis. Am J Chin Med 2008;36:675-83.

31. Ji H, Yao S, Luo Q, et al. TCM treatment of rheumatoid arthritis by supplementing the kidney and invigorating the blood circulation. $J$ Tradit Chin Med 2002;22:252-5.

32. Jiang $Q$, Zhou $X Y$, Wang $L$, et al. A one-year evaluation of radiographic progression in patients with rheumatoid arthritis treated by Qingre Huoxue Decoction (). Chin J Integr Med 2012;18:256-61.

33. Jiao J, Jiang Q. [External application of compound Tripterygium wilfordii decreased the activity of rheumatoid arthritis]. Zhongguo Zhong Xi Yi Jie He Za Zhi 2012;32:1470-2, 76.

34. Jie HY, Wu QF, Ding ZX. [Clinical study of Biqi Capsule combined with methotrexate for treatment of rheumatoid arthritis]. Zhongguo Zhong Xi Yi Jie He Za Zhi 2012;32:195-8.

35. Kang XZ, Wu QF, Wang KH. [Efficacy of integrative medicine for the treatment of rheumatoid arthritis and its effect on glucocorticoid receptor expression]. Zhongguo Zhong Xi Yi Jie He Za Zhi 2010;30:1261-4.

36. Li EK, Tam LS, Wong CK, et al. Safety and efficacy of Ganoderma lucidum (lingzhi) and San Miao San supplementation in patients with rheumatoid arthritis: a double-blind, randomized, placebo-controlled pilot trial. Arthritis Rheum 2007;57:1143-50.

37. Li YN, Zhao YS, Li X. [Study on abnormality and regulation of T-lymphocyte subsets in peripheral blood of rheumatoid arthritis patients]. Zhongguo Zhong Xi Yi Jie He Za Zhi 2002;22: 359-61.

38. Li YS, Tong PJ, Ma HZ. [Toxicity attenuation and efficacy potentiation effect of liquorice on treatment of rheumatoid arthritis 
with Tripterygium wilfordii]. Zhongguo Zhong Xi Yi Jie He Za Zhi 2006;26:1117-19.

39. Liang $\mathrm{Q}$, Tang $\mathrm{T}$, Zhang $\mathrm{H}$. [Clinical investigation of effects of bizhongxiao decoction (BZX) on rheumatoid arthritis on active phase]. Hunan Yi Ke Da Xue Xue Bao 2000;25:449-52.

40. Lin CS, Yang XY, Dai L. [Multi-center clinical study on therapeutic effect of kunxian capsule on rheumatoid arthritis]. Zhongguo Zhong Xi Yi Jie He Za Zhi 2011;31:769-74.

41. Liu DF, Guo MY, Zhang J. [Treatment of active rheumatoid arthritis by sanhuang yilong decoction]. Zhongguo Zhong Xi Yi Jie He Za Zhi 2008;28:743-6.

42. Liu J, Li H, Chen X. [Effects of traditional Chinese medicine for invigorating spleen to resolve dampness and dredging collaterals on patients with rheumatoid arthritis and anemia]. Zhong Xi Yi Jie He Xue Bao 2006;4:348-54.

43. Liu W, Liu XY, Liu B. [Clinical observation on treatment of rheumatoid arthritis with zhengqing fengtongning retard tablets: a report of 60 cases]. Zhong Xi Yi Jie He Xue Bao 2006;4:201-2.

44. Liu W, Liu XY, Wang Y. [Effect of Chinese herbs in enhancing prednisone for treatment of refractory rheumatoid arthritis] Zhongguo Zhong Xi Yi Jie He Za Zhi 2007;27:742-4.

45. Liu W, Zhang L, Xu Z. [Clinical observation on treatment of rheumatoid arthritis with biqi capsule]. Zhongguo Zhong Xi Yi Jie He Za Zhi 2006;26:157-9.

46. Liu XD, Zhang JL, Ye LH. [Effects of wenhua juanbi recipe on TNF-alpha and IL-1beta in peripheral blood of rheumatoid arthritis patients]. Zhongguo Zhong Xi Yi Jie He Za Zhi 2009;29:787-90.

47. Lu SJ, Shao J, Li XR. [Clinical observation on treatment of rheumatoid arthritis by combined therapy with methotrexate, sulfasalazine and Chinese herbal medicine]. Zhongguo Zhong Xi Yi Jie He Za Zhi 2002;22:571-3.

48. Shen HB, Bai YJ, Huo ZJ, et al. Assessment of clinical effect of therapy combining disease with syndrome on rheumatoid arthritis. $J$ Tradit Chin Med 2011:31:39-43.

49. Shen $Y, Q u Q$, Wang $D$. Clinical observation on treatment of rheumatoid arthritis with fengshi xiandan in 53 cases. J Tradit Chin Med 2003;23:21-6.

50. Song YW, Lee EY, Koh E-M, et al. Assessment of comparative pain relief and tolerability of SKI306X compared with celecoxib in patients with rheumatoid arthritis: a 6-week, multicenter, randomized, double-blind, double-dummy, phase III, noninferiority clinical trial. Clin Ther 2007;29:862-73.

51. Tao X, Younger J, Fan FZ, et al. Benefit of an extract of Tripterygium Wilfordii Hook $\mathrm{F}$ in patients with rheumatoid arthritis: a double-blind, placebo-controlled study. Arthritis Rheum 2002;46:1735-43.

52. Wang JM, Tao QW, Zhang $\mathrm{YZ}$, et al. [Treating rheumatoid arthritis patients of Shen deficiency and cold invading syndrome by bushen quhan zhiwang decoction combined methotrexate: an evaluation of clinical efficacy and safety]. Zhongguo Zhong Xi Yi Jie He Za Zhi 2013;33:614-18.

53. Wu YJ, Lao ZY, Zhang ZL. [Clinical observation on small doses Tripterygium wilfordii polyglycoside combined with methotrexate in treating rheumatoid arthritis]. Zhongguo Zhong Xi Yi Jie He Za Zhi 2001;21:895-6.

54. Yang W, Ouyang J, Zhu K, et al. TCM treatment for 40 cases of rheumatoid arthritis with channel blockage due to yin deficiency. $J$ Tradit Chin Med 2003;23:172-4.

55. Yu JP, Zhang YZ, Li XY. [Effect of strengthening Pi and activating blood circulation therapy on serum levels of adrenocorticotrophic hormone and vascular endothelial growth factor in rheumatoid arthritis patients]. Zhongguo Zhong Xi Yi Jie He Za Zhi 2007:27:593-5.

56. Yu SY, Yu ZA. [Clinical observation of compound xiatianwu tablets in treatment of 120 cases with active rheumatoid arthritis]. Zhongguo Zhong Yao Za Zhi 2013;38:899-901.

57. Yu WY, Shen SW, Yang ZH. A clinical study of Suogudan granule in the treatment of rheumatoid arthritis. Chin $\mathrm{J}$ Integr Med 2005;11:255-9.

58. Zhang JH, Wang JP, Wang HJ. [Clinical study on effect of total panax notoginseng saponins on immune related inner environment imbalance in rheumatoid arthritis patients]. Zhongguo Zhong Xi Yi Jie He Za Zhi 2007;27:589-92.

59. Zhao SS, Wang J. [Short-term clinical observation on compound Xiatianwu combined with methotrexate in treating rheumatoid arthritis]. Zhongguo Zhong Yao Za Zhi 2012;37:3664-6.

60. Zhao YX, Liu Y. [Clinical observation on effects of leflunomid and total glucosides of paeony on rheumatoid arthritis]. Zhongguo Zhong Xi Yi Jie He Za Zhi 2006;26:355-7.

61. Zhou CY, Pan Z, Ma F, et al. [Controlled clinical trials of external using of Cheezheng Qingpeng ointment on reliving analgesia and swelling for the treatment of rheumatoid arthritis]. Zhongguo Gu Shang 2009;22:917-19.

62. Zhou CY, Tang JY, Fang DY. [Clinical study on active rheumatoid arthritis treated with simiao xiaobi decoction]. Zhongguo Zhong $X$ Yi Jie He Za Zhi 2010;30:275-9.

63. Zhou DQ, Liu DX. [Juanbi mixture in treating 100 cases of rheumatoid arthritis patients]. Zhongguo Zhong Xi Yi Jie He Za Zhi 2002;22:386-7.

64. Zhou H, Zhong Y. [Clinical observation on liang's anti-rheumatism and rheumatoid granule in rheumatoid arthritis in the active stage] Zhongguo Zhong Xi Yi Jie He Za Zhi 2006;26:248-50.

65. Zhou X, Zhou Z, Jin M, et al. Intermediate and late rheumatoid arthritis treated by tonifying the kidney, resolving phlegm and removing blood stasis. J Tradit Chin Med 2000;20:87-92.

66. Zhou X, Zhou Z, Jin M, et al. Clinical study of qingluo tongbi granules in treating 63 patients with rheumatoid arthritis of the type of yin-deficiency and heat in collaterals. J Tradit Chin Med 2004;24:83-7.

67. Zhang GX, Zhang YM, Cao SY. Ameliorating effect of externa therapy of Chinese herb on joint function of patients with rheumatoid arthritis. Chinese Journal of Clinical Rehabilitation 2006;10:24-6.

68. Zuo C, Chen YT, Wang ZM. [Clinical efficacy of Corydalis composite combined with methotrexate in treating rheumatoid arthritis]. Zhongguo Zhong xi yi jie he za zhi Zhongguo Zhongxiyi jiehe zazhi=Chinese journal of integrated traditional and Western medicine / Zhongguo Zhong xi yi jie he xue hui, Zhongguo Zhong yi yan jiu yuan zhu ban 2009;29:1023-5.

69. Sun SM, Min SP, Luo SP, et al. Clinical study on effect of Tongyong Tongfeng Decoction combined with methotrexate on pain and swelling after rheumatoid arthritis. Chinese Journal of Clinical Rehabilitation 2002;6:148-9.

70. Li HR, Zhang X, Ding J, et al. Curative effect of Yishen Juanbi Capsule combined with fumigation therapy by Chinese materia medica on rheumatoid arthritis. Chinese Traditional and Herbal Drugs 2013;44:335-7.

71. Hang WP, Li J, Xu J, et al. Double-blind randomized placebo controlled trial (phase II) on the efficacy and safety of Ranjiangduoj capsule in the treatment of patients with active rheumatoid arthritis (Rtention of Cold-damp in the collaterals). Chinese Journal of Evidence-Based Medicine 2005;5:286-90+322.

72. Xun XQ, Xiong XG, Chen J, et al. Effects of heat-clearing and detoxicating (bizhongxiao decoction) on bone erosion of rheumatoid arthritis: compared with combination of methotrexate and sufasalazine therapy. Journal of Clinical Rehabilitative Tissue Engineering Research 2010;14:7696-9.

73. Ding F, Zhang Y, Chang Y. Effects of Sanwushaoxie decoction on IL-18, TNF-alpha, IL-4 and IL-10 in patients with rheumatoid arthritis. Zhongguo Zhongyao Zazhi 2010;35:790-3.

74. Tang SJ, Yue YS, Zhao WH. 53 cases of rheumatoid arthritis treated with quartet therapy. Journal of Anhui Traditional Chinese Medical College 2002. http://onlinelibrary.wiley.com/o/cochrane/ clcentral/articles/811/CN-00716811/frame.html

75. Tu SH, Hu YH. [Assessment on life quality of patients with rheumatoid arthritis and efficacy of tripterygium wilfordii hook]. Journal of Traditional Chinese Medicine University of Hunan [hu Nan Zhong Yi Xue Yuan Xue Bao] 2006. http://onlinelibrary.wiley. com/o/cochrane/clcentral/articles/193/CN-00976193/frame.html

76. Guo JB, Xue G, Zhang LF, et al. [Clinical observation of treating rheumatoid arthritis using compound tripterygium wilfordii wine]. Hebei Journal of Traditional Chinese Medicine [he Bei Zhong Yi] 2003. http://onlinelibrary.wiley.com/o/cochrane/clcentral/articles/302/ $\mathrm{CN}-00975302 /$ frame.html

77. Chen ZH. [Clinical observation of Yishen Zhuanggu Tongbi Decoction in the treatment of rheumatoid arthritis]. Journal of Guangzhou University of Traditional Chinese Medicine [guang Zhou Zhong Yi Yao da Xue Xue Bao] 2005. http://onlinelibrary.wiley.com/ o/cochrane/clcentral/articles/005/CN-00976005/frame.html

78. Guan BS. [Clinical observation on the effect of acupuncture combined with Chinese medicine for rheumatoid arthritis]. Chinese Journal of Gerontology [zhong Guo Lao Nian Xue za Zhi] 2005. http://onlinelibrary.wiley.com/o/cochrane/clcentral/articles/015/ $\mathrm{CN}-00976015 /$ frame.html

79. Ma HY, Huo YL, Ma XS. [Clinical observation on the effect of acupunture combined with Chinese medicine for rheumatoid arthritis]. Clinical Journal of Traditional Chinese Medicine [zhong Yi Yao Lin Chuang za Zhi] 2008. http://onlinelibrary.wiley.com/o/ cochrane/clcentral/articles/783/CN-00976783/frame.htm

80. Hu YM. [Clinical observation on the effect of Chinese medicine combined with anus suppository for rheumatoid arthritis in 74 cases]. Zhejiang Clinical Medical Journal [zhe Jiang Lin Chuang Yi 
Xue] 2007. http://onlinelibrary.wiley.com/o/cochrane/clcentral/ articles/391/CN-00976391/frame.htm

81. Li WJ, Liu P. [Clinical observation on the effect of Chinese medicine for rheumatoid arthritis]. Chinese Journal of Traditional Medical Traumatology \& Orthopedics [zhong Guo Zhong Yi Gu Shang] 2003. http://onlinelibrary. wiley.com/o/cochrane/clcentral/ articles/316/CN-00975316/frame.htm

82. Liu YH, Ren XR, Zhang QX, et al. [Clinical observation on the effect of Chinese medicine, physical therapy and exercises for rheumatoid arthritis in 50 cases]. Modern Rehabilitation [xian Dai Kang Fu] 2000. http://onlinelibrary.wiley.com/o/cochrane/clcentral/ articles/089/CN-00975089/frame.html

83. Wang ZQ. [Clinical observation on the effect of external and internal use of Chinese medicine for rheumatoid arthritis]. Hubei Journal of Chinese Medicine [bu Bei Zhong Yi za Zhi] 2001. http:/l onlinelibrary.wiley.com/o/cochrane/clcentral/articles/090/ CN-00975090/frame.html

84. Xiao M. [Clinical observation on the effect of Fengshi Tincture for rheumatoid arthritis in 48 cases]. Journal of Baotou Medical College [bao Tou Yi Xue Yuan Xue Bao] 2008. http://onlinelibrary. wiley.com/o/cochrane/clcentral/articles/778/CN-00976778/frame. html

85. Zou YJ. [Clinical observation on the effect of Guizhi Shaoyao Zhimu Decoction for rheumatoid arthritis in 30 cases]. Journal of Emergency in Traditional Chinese Medicine [zhong Guo Zhong Yi Ji Zheng] 2007. http://onlinelibrary.wiley.com/o/cochrane/clcentral/ articles/372/CN-00976372/frame.htm

86. Wang SM, Wang X, Gong Q. [Clinical observation on the effect of integrated Chinese and western medicine for rheumatoid arthritis in 40 cases]. Journal of Sichuan of Traditional Chinese Medicine[si Chuan Zhong Yi] 2006. http://onlinelibrary.wiley.com/o/cochrane/ clcentral/articles/208/CN-00976208/frame.htm

87. Ma YB, Li DM, Zhang HD. [Clinical observation on the effect of self-developed Fengshiling Wine for rheumatoid arthritis]. Chinese Journal of Convalescent Medicine [zhong Guo Liao Yang Yi Xue] 2002. http://onlinelibrary.wiley.com/o/cochrane/clcentral/articles/093/ $\mathrm{CN}-00975093 /$ frame.htm

88. Wu CL. [Clinical observation on the effect of Siwu Wuteng Decoction combined with methylamine pterin for rheumatoid arthritis in 46 cases]. Jiangxi Journal of Traditinal Chinese Medicine 2004. http://onlinelibrary.wiley.com/o/cochrane/clcentral/articles/789/ CN-00975789/frame htm

89. Jiang GL, Liu DJ, Wang FG, et al. [Clinical observation on the effect of Tongbi Pills combined with Wutou Capsules for rheumatoid arthritis]. Acta Chinese Medicine and Pharmacology [Zhong Yi Yao Xue Bao] 2000. http://onlinelibrary.wiley.com/o/ cochrane/clcentral/articles/076/CN-00975076/frame.htm

90. Liang YM. [Clinical observation on the effect of Yishen Huoxue Juanbi Decoction for rheumatoid arthritis in 36 cases]. Yunnan Journal of Traditional Chinese Medicine and Materia Medica [yun Nan Zhong Yi Zhong Yao za Zhi] 2000. http://onlinelibrary.wiley. com/o/cochrane/clcentral/articles/095/CN-00975095/frame.htm

91. Tao JZ. [Clinical observation on the effect of Zhuifeng Tougu Huoluo Pell combined with Zhuifeng Tougu Huoluo Powder for rheumatoid arthritis in 70 cases]. Chinese Community Doctors [zhong Guo She Qu Yi Shi] 2002. http://onlinelibrary.wiley.com/o/ cochrane/clcentral/articles/092/CN-00975092/frame.html

92. Lv MH, Zhang YQ. [Clinical observation on the long term effect of Juanbi Decoction combined with Huangqi injection for rheumatoid arthritis]. Anhui Clinical Journal of Traditional Chinese Medicine [an Hui Zhong Yi Lin Chuang za Zhi] 2000. http://onlinelibrary.wiley. com/o/cochrane/clcentral/articles/063/CN-00975063/frame.html

93. Zhang GH. [Clinical research on the effect of Shirebi Compound for rheumatoid arthritis]. Hubei Journal of Traditional Chinese Medicine [hu Bei Zhong Yi za Zhi] 2005. http://onlinelibrary.wiley.com/o/ cochrane/clcentral/articles/998/CN-00975998/frame.htm

94. Chen X, Li H. [Clinical research on the effect of Zhengqing Fengtongning for rheumatoid arthritis]. Journal of Anhui Traditional Chinese Medicine College [an Hui Zhong Yi Xue Yuan Xue Bao] 2005. http://onlinelibrary.wiley.com/o/cochrane/clcentral/articles/018/ CN-00976018/frame.html

95. Xiao F, Wu FJ. [Clinical study of Bi Qi Capsule for rheumatoid arthritis]. Journal of Liaoning University of Traditional Chinese Medicine [liao Ning Zhong Yi Yao da Xue Xue Bao] 2008. http:// onlinelibrary.wiley.com/o/cochrane/clcentral/articles/748/ $\mathrm{CN}-00976748 /$ frame.html

96. Huang GD, Li JB, Huang YH, et al. [The clinical study on sinomenine for 100 patients with rheumatoid arthritis]. Journal of Emergency in Traditional Chinese Medicine [zhong Guo Zhong Yi Ji Zheng] 2007. http://onlinelibrary.wiley.com/o/cochrane/clcentra// articles/380/CN-00976380/frame.html
97. Fan $\mathrm{CP}$, Chen Q, Qi WL. [Comparison of Zhengqing Fengtongning and tripterygium glycosides for rheumatoid arthritis]. China Pharmaceuticals [zhong Guo Yao Ye] 2000. http://onlinelibrary. wiley.com/o/cochrane/clcentral/articles/096/CN-00975096/frame. html

98. Zhen HX, Liu W, Zhang L. [Curative effect of combined Chinese medicines on rheumatoid arthritis with damp-heat blocking collaterals]. Tianjin Journal of Traditional Chinese Medicine [tian Jin Zhong Yi Yao] 2006. http://onlinelibrary.wiley.com/o/cochrane/ clcentral/articles/207/CN-00976207/frame.html

99. Ji YL, Wan WW, Chen Y, et al. [Curative effect of external application of Chinese medicine on active rheumatoid arthritis]. Journal of Nursing Science [hu Li Xue za Zhi] 2008. http:// onlinelibrary.wiley.com/o/cochrane/clcentral/articles/785/ $\mathrm{CN}-00976785 /$ frame.html

100. Xu DS, Tong LM, Qin DH, et al. [Effect of Wudang Zhuifeng Shengshi Liquor as fumigation bath for ESR, CRP and RF in rheumatoid arthritis]. Hubei Journal of Traditional Chinese Medicine [hu Bei Zhong Yi za Zhi] 2005. http://onlinelibrary.wiley.com/o/ cochrane/clcentral/articles/011/CN-00976011/frame.htm

101. Wang $\mathrm{Y}, \mathrm{Wu} \mathrm{Q}$, Wei JX, et al. [Experience of total glucosides of paeony, Methotrexate and Tripterygium wilfordii(Tripterygium Wilfordii Hook) for rheumatoid arthritis in 150 cases]. Journal of Zhengzhou University(medical Sciences) [zhen Zhou da Xue Xue Bao (yi Xue Ban)] 2006. http://onlinelibrary. wiley.com/o/cochrane/ clcentral/articles/186/CN-00976186/frame.html

102. Liu L. [The internal and external use of Feng Shi Ning Decoction for rheumatoid arthritis in 32 cases]. Shaanxi Jornal of Traditional Chinese Medicine [shan Xi Zhong Yi] 2007. http://onlinelibrary.wiley. com/o/cochrane/clcentral/articles/370/CN-00976370/frame.html

103. He JF, Liang YY. [Observation of rheumatoid arthritic treated by integration of traditional Chinese medicine and western medicine] Hebei Journal of Traditional Chinese Medicine [he Bei Zhong Yi] 2001. http://onlinelibrary.wiley.com/o/cochrane/clcentral/articles/088/ CN-00975088/frame.html

104. Liu XY, Liu JH, Liu LN, et al. [Observation of rheumatoid arthritis treated by a traditional Chinese medicine method of removing blood stasis and invigorating for deficiency]. Hebei Journal of Traditional Chinese Medicine [he Bei Zhong Yi] 2003. http:/l onlinelibrary.wiley.com/o/cochrane/clcentral/articles/299/ $\mathrm{CN}-00975299 /$ frame.html

105. Huang N, Huang CJ, Huang XF. [Observation of Rheumatoid arthritis treated by Sanbi decoction]. Hebei Journal of Traditional Chinese Medicine [he Bei Zhong Yi] 2002. http://onlinelibrary.wiley. com/o/cochrane/clcentral/articles/073/CN-00975073/frame.html

106. $\mathrm{Li} \mathrm{B}, \mathrm{Li}$ Y. [Treatment of early rheumatoid arthritis wiht Chinese medicine combined with acupuncture in 46 cases]. Shandong Medical Journal [shan Dong Yi Yao] 2001. http://onlinelibrary.wiley. com/o/cochrane/clcentral/articles/091/CN-00975091/frame.html

107. Li JC, Zhao DR. [Treatment of refractory rheumatoid arthritis with integrated Chinese and western medicine in 86 cases]. Journal of Sichuan of Traditional Chinese Medicine[si Chuan Zhong Yi] 2003. http://onlinelibrary.wiley.com/o/cochrane/clcentral/articles/319/ CN-00975319/frame.html

108. Xin XF, Chen Y. [Treatment of rheumatoid arthritis in old age with integrated Chinese and western medicine]. Zhejiang Journal of Integrated Traditional Chinese and Western Medicine [zhe Jiang Zhong Xi Yi Jie He za Zhi] 2005. http://onlinelibrary.wiley.com/o/ cochrane/clcentral/articles/007/CN-00976007/frame.htm

109. Leng YL, Li Y, Chen XN, et al. [Treatment of rheumatoid arthritis with acupoint thread implanting combined with Chinese medicine in 48 cases]. Lishizhen Medicine and Materia Medica Research [shi Zhen Guo Yi Guo Yao] 2000. http://onlinelibrary.wiley.com/o/ cochrane/clcentral/articles/082/CN-00975082/frame.htm

110. Wang YG, Song JY, Zhao T. [Treatment of rheumatoid arthritis with Chinese medicine combined with multi-function inotophoresis in 108 cases]. China Science and Technology Information [zhong Guo Ke Ji Xin Xe] 2005. http://onlinelibrary.wiley.com/o/cochrane/ clcentral/articles/009/CN-00976009/frame.html

111. Zeng $\mathrm{YH}$, Huang RQ. Treatment of rheumatoid arthritis with Feng Shi Ning Injection in 42 cases. Chinese Journal of Information on TCM 2006. http://onlinelibrary.wiley.com/o/cochrane/clcentral/ articles/980/CN-00796980/frame.htm

112. Ji FR, Zhao XL. [Treatment of rheumatoid arthritis with Guizhi Shaoyao Zhimu Decoction in 45 cases]. Chinese Community Doctors [zhong Guo She Qu Yi Shi] 2007. http://onlinelibrary.wiley. com/o/cochrane/clcentral/articles/371/CN-00976371/frame.htm

113. Cai JW, Hong BF. [Treatment of rheumatoid arthritis with Guizhi Shaoyao Zhimu Decoction in 50 cases]. Gansu Journal of Traditional Chinese Medicine 2006. http://onlinelibrary.wiley.com/o/ cochrane/clcentral/articles/190/CN-00976190/frame.html 
114. Wu JR, Meng LH. [Treatment of rheumatoid arthritis with integrated Chinese and western medicine in 56 cases]. Practical Clinical Journal of Integrated Traditional Chinese and Western Medicine[shi Yong Zhong Xi Yi Jie He Lin Chuang] 2004. http://onlinelibrary. wiley. com/o/cochrane/clcentral/articles/797/CN-00975797/frame.html

115. Guo SX. [Treatment of rheumatoid arthritis with modified Baihu Guizhi Tang in 120 cases]. Modern Journal of Integrated Traditional Chinese and Western Medicine [xian Dai Zhong Xi Yi Jie He za Zhi] 2003. http://onlinelibrary.wiley.com/o/cochrane/ clcentral/articles/297/CN-00975297/frame.html

116. Li F, Pang XF. [Treatment of rheumatoid arthritis with Sanbi Decoction in 30 cases]. Shaanxi Journal of Traditional Chinese Medicine [shan Xi Zhong Yi] 2008. http://onlinelibrary.wiley.com/o/ cochrane/clcentral/articles/770/CN-00976770/frame.html

117. Chen YL. [Treatment of rheumatoid arthritis with self-developed Huangqi Shudi Jisheng Decoction in 140 cases]. Shaanxi Journal of Traditional Chinese Medicine [shan Xi Zhong Yi] 2007. http:/ onlinelibrary.wiley.com/o/cochrane/clcentral/articles/395/ CN-00976395/frame.html

118. Li GJ, Bai CY, Wang B, et al. [Treatment of rheumatoid arthritis with self-developed Juanbi Decoction combined with MTX in 24 cases]. Chinese Journal of Integrated Traditional and Western Medicine [zhong Guo Zhong Xi Yi Jie He za Zhi] 2003. http:/l onlinelibrary.wiley.com/o/cochrane/clcentral/articles/317/ CN-00975317/frame.html

119. Du YK, Wang JW, Huang SQ. [Treatment of rheumatoid arthritis with self-developed Sancao Juanbi Decoction in 40 cases]. Modern Traditional Chinese Medicine[xian Dai Zhong Yi Yao] 2003. http:// onlinelibrary.wiley.com/o/cochrane/clcentral/articles/318/ $\mathrm{CN}-00975318 /$ frame.html

120. Xu C-H. [Treatment of rheumatoid arthritis with warm needling and Juanbi decoction:A report of 35 cases]. Journal of Anhui Traditional Chinese Medical College [An Hui Zhong Yi Xue Yuan Xue Bao] 2004. http://onlinelibrary.wiley.com/o/cochrane/clcentral/articles/583/ CN-00676583/frame.html

121. Shi B. [Treatment of rheumatoid arthritis with Wutou Decoction in 64 cases]. Chinese Community Doctors [zhong Guo She Qu Yi Shi] 2007. http://onlinelibrary.wiley.com/o/cochrane/clcentral/ articles/386/CN-00976386/frame.html

122. Hui CL, Li FP. [Treatment of rheumatoid arthritis with Yanghe Decoction combined with tripterygium glycosides in 60 cases]. Shaanxi Journal of Traditional Chinese Medicine [shan Xi Zhong Yi] 2008. http://onlinelibrary.wiley.com/o/cochrane/clcentral/articles/ 780/CN-00976780/frame.html

123. Meng QL, Guo HQ, Zhao YQ. [Treatment of rheumatoid arthritis with Yishen Juanbi Decoction combined with MTX in 40 cases]. Traditional Chinese Medicine Research [zhong Yi Yan Jiu] 2006. http://onlinelibrary.wiley.com/o/cochrane/clcentral/articles/205/ CN-00976205/frame.html

124. Wang W. [Xianteng Fengshi Decoction in treating rheumatoid arthritis]. Journal of Henan University of Chinese Medicine[he Nan Zhong Yi Xue Yuan Xue Bao] 2004. http://onlinelibrary.wiley.com/o/ cochrane/clcentral/articles/794/CN-00975794/frame.html

125. Huang CB, Liu J, Chen X, et al. [Treatment of rheumatoid arthritis by xinfeng capsule: an efficacy observation]. Zhongguo Zhong Xi Yi Jie He Za Zhi 2013;33:1599-602.

126. Kou QA, Li L, Yao JY, et al. [Treatment of rheumatoid arthritis arthralgia by xiaoyan zhitong paste: a clinical observation]. Zhongguo Zhong Xi Yi Jie He Za Zhi 2013;33:1320-2, 27.

127. Liu J, Cao Y, Huang C, et al. Use of xinfeng capsule to treat abarticular pathologic changes in patients with rheumatoid arthritis. $J$ Tradit Chin Med 2014;34:532-8.

128. Wang Z, Tao XJ. [Treatment of rheumatoid arthritis by Yangxue Tongluo Recipe combined with immunosuppressive agents: a clinical observation]. Zhongguo Zhong Xi Yi Jie He Za Zhi 2014;34:276-8.

129. Guo SY, Lin LJ, Chu H, et al. [Efficacy of Lugua polypeptide injection on active rheumatoid arthritis]. Zhongguo Gu Shang 2008;21:610-12.

130. Tao Q, Xu Y, Jin D, et al. Clinical effect and safety of Lugua Polypeptide injection in the treatment of rheumatoid arthritis. Journal of Clinical Orthopaedics 2011;14:336-8.

131. Zhu Y, Lin W, Yang F, et al. Lugua Polypeptide injection in the treatment of rheumatoid arthritis. Journal of Clinical Orthopaedics 2011;14:334-6.

132. Zheng $\mathrm{LH}$, Kang $\mathrm{XZ}$, Wang $\mathrm{KH}$, et al. Clinical research of the syndrome differentiation treatment of TCM in combination with stage-based treatment of western medicine on patients with rheumatoid arthritis. JETCM 2009;18:1256-8.

133. Liu J, Liu X, Han M. Treatment of 20 cases of rheumatoid arthritis in active stage with Xinfeng capsule. Journal of Anhui Traditional Chinese Medical College 2003;22:12-16.
134. Sun S, Zhou C, Fan B. Clinical study on Bisuqing Mixture for rheumatoid arthritis in active stage. Shandong J Tradit Chin Med 2003;2003:9.

135. Cui Z, Bian R, Chen Z, et al. Clinical observation of Bitong decoction treating 54 cases of rheumatoid arthritis. Journal of Sichuan of Traditional Chinese Medicine[si Chuan Zhong Yi] 2004;22:39-40.

136. Liu J, Wang C, Liu S. Clinical research of YiShenJuanBi capsules in the treatment of rheumatoid arthritis. Chinese $J$ Trad Med Traum \& Orthop 2008;16:24-6.

137. Ao X, Chen $\mathrm{C}$, Zen $\mathrm{H}$, et al. Synergistic action of fuming - washing therapy with Qingbixifang on rheumatoid arthritis with heat retention. Journal of Guangzhou University of Traditional Chinese Medicine [guang Zhou Zhong Yi Yao da Xue Xue Bao] 2005;22:436-9.

138. Wu Y, Lao Z, Zhang Z. Clinical observation on small doses tripterygium wilfordii polyglycoside combined with methotrexate in treating rheumatoid arthritis. Chinese Journal of Integrated Traditional and Western Medicine [zhong Guo Zhong Xi Yi Jie He za Zhi] 2001;21:895.

139. Wang D. Clinical observation on combine with total Glucosides of Peony in treating 32 cases rheumatoid arthritis. Shaanxi Journal of Traditional Chinese Medicine [shan Xi Zhong Yi] 2007;28:421-2.

140. He D, Shen J, Zhang Z, et al. "Shi Re Bi Granule" for rheumatoid arthritis in 46 cases. Shanghai Journal of Traditional Chinese Medicine 2002;36:14-15.

141. Lv QW, Zhang W, Shi Q, et al. Comparison of Tripterygium wilfordii Hook $F$ with methotrexate in the treatment of active rheumatoid arthritis (TRIFRA): a randomised, controlled clinical trial. Ann Rheum Dis 2014.

142. Tai-xiang W, You-ping L, Zhao-xiang B, et al. Consolidated Standards for Reporting Trials of Traditional Chinese Medicine (CONSORT for TCM). Chinese Journal of Evidence-Based Medicine 2007;8:601-5.

143. Prevoo ML, van 't Hof MA, Kuper HH, et al. Modified disease activity scores that include twenty-eight-joint counts. Development and validation in a prospective longitudinal study of patients with rheumatoid arthritis. Arthritis Rheum 1995;38:44-8.

144. van der Heijde DM, van 't Hof M, van Riel PL, et al. Development of a disease activity score based on judgment in clinical practice by rheumatologists. J Rheumatol 1993;20:579-81.

145. Felson DT, Anderson JJ, Boers M, et al. American College of Rheumatology. Preliminary definition of improvement in rheumatoid arthritis. Arthritis Rheum 1995:38:727-35.

146. van Gestel AM, Prevoo ML, van 't Hof MA, et al. Development and validation of the European League Against Rheumatism response criteria for rheumatoid arthritis. Comparison with the preliminary American College of Rheumatology and the World Health Organization/International League Against Rheumatism Criteria. Arthritis Rheum 1996;39:34-40.

147. Arnett FC, Edworthy SM, Bloch DA, et al. The American Rheumatism Association 1987 revised criteria for the classification of rheumatoid arthritis. Arthritis Rheum 1988;31:315-24.

148. Aletaha D, Neogi T, Silman AJ, et al. 2010 rheumatoid arthritis classification criteria: an American College of Rheumatology/ European League Against Rheumatism collaborative initiative. Ann Rheum Dis 2010;69:1580-8.

149. Bykerk VP, Lie E, Bartlett SJ, et al. Establishing a core domain set to measure rheumatoid arthritis flares: report of the OMERACT 11 RA flare Workshop. J Rheumatol 2014;41:799-809.

150. Kim YW, Zhang MQ. Characteristics of traditional Chinese medicine in treating rheumatoid arthritis: a retrospective analysis. Chinese Journal of Clinical Rehabilitation 2005:9:152-5.

151. Zhang C, Jiang M, Lu A. Chinese herbal medicines versus disease modifying antirheumatic drugs for management of rheumatoid arthritis: a systematic review. Eur J Integr Med 2011;3:e219-31.

152. Cameron M, Gagnier JJ, Chrubasik S. Herbal therapy for treating rheumatoid arthritis. Cochrane Database Syst Rev 2011;(2):CD002948.

153. Jiang M, Yang J, Zhang C, et al. Clinical studies with traditional Chinese medicine in the past decade and future research and development. Planta Med 2010;76:2048-64.

154. Jadad AR, Moore RA, Carroll D, et al. Assessing the quality of reports of randomized clinical trials: is blinding necessary? Control Clin Trials 1996;17:1-12.

155. Schulz KF, Chalmers I, Hayes RJ, et al. Empirical evidence of bias. Dimensions of methodological quality associated with estimates of treatment effects in controlled trials. JAMA 1995;273:408-12.

156. Califf RM, Zarin DA, Kramer JM, et al. Characteristics of clinical trials registered in ClinicalTrials.gov, 2007-2010. JAMA 2012;307:1838-47.

157. Wu XY, Tang JL, Mao C, et al. Systematic reviews and meta-analyses of traditional Chinese medicine must search Chinese databases to reduce language bias. Evid Based Complement Alternat Med 2013;2013:812179. 